Supplementary information

\title{
A Train of Single Molecule-Gears
}

We - Hyo Soe ${ }^{\dagger \dagger}$ Saurabh Srivastava, ${ }^{\dagger}$ and Christian Joachim ${ }^{\dagger \dagger}$

†Centre d'Elaboration de Matériaux et d'Études Structurales (CEMES), Centre National de la Recherche Scientifique (CNRS), Université de Toulouse, 29 Rue J. Marvig, BP 4347, 31055 Toulouse Cedex, France

*International Center for Materials Nanoarchitectonics (WPI-MANA), National Institute for Material Sciences (NIMS), 1-1 Namiki, Tsukuba, Ibaraki 305-0044, Japan

\section{Contents}

Suppl. Info. S1: Molecule-gears on a $\mathrm{Pb}(111)$ surface

Suppl. Info. S2: Atomic scale axle height

Suppl. Info. S3: Molecular mechanics calculations of the handle effect.

Suppl. Info. S4: Direct STM mechanical manipulation of a single molecule-gear

Suppl. Info. S5: Rotational manipulation of a molecule-gear by voltage pulse

Suppl. Info. S6: Movies

Suppl. Info. S7: Ripping of the rotation transmission with only 2 molecule-gears 


\section{Suppl. Info. S1: Molecule-gears on a $\mathrm{Pb}(111)$ surface}

As presented in Fig. $\mathrm{S} 1$ below, most of the $\mathrm{C}_{64} \mathrm{~N}_{2} \mathrm{H}_{76}$ molecules sublimated on the $\mathrm{Pb}(111)$ surface with the $\mathrm{Pb}(111)$ surface kept at room temperature are stabilized forming $2 \mathrm{D}$ islands on small terraces in between mono-atomic step edges. A few molecules were also found stabilized around impurities.
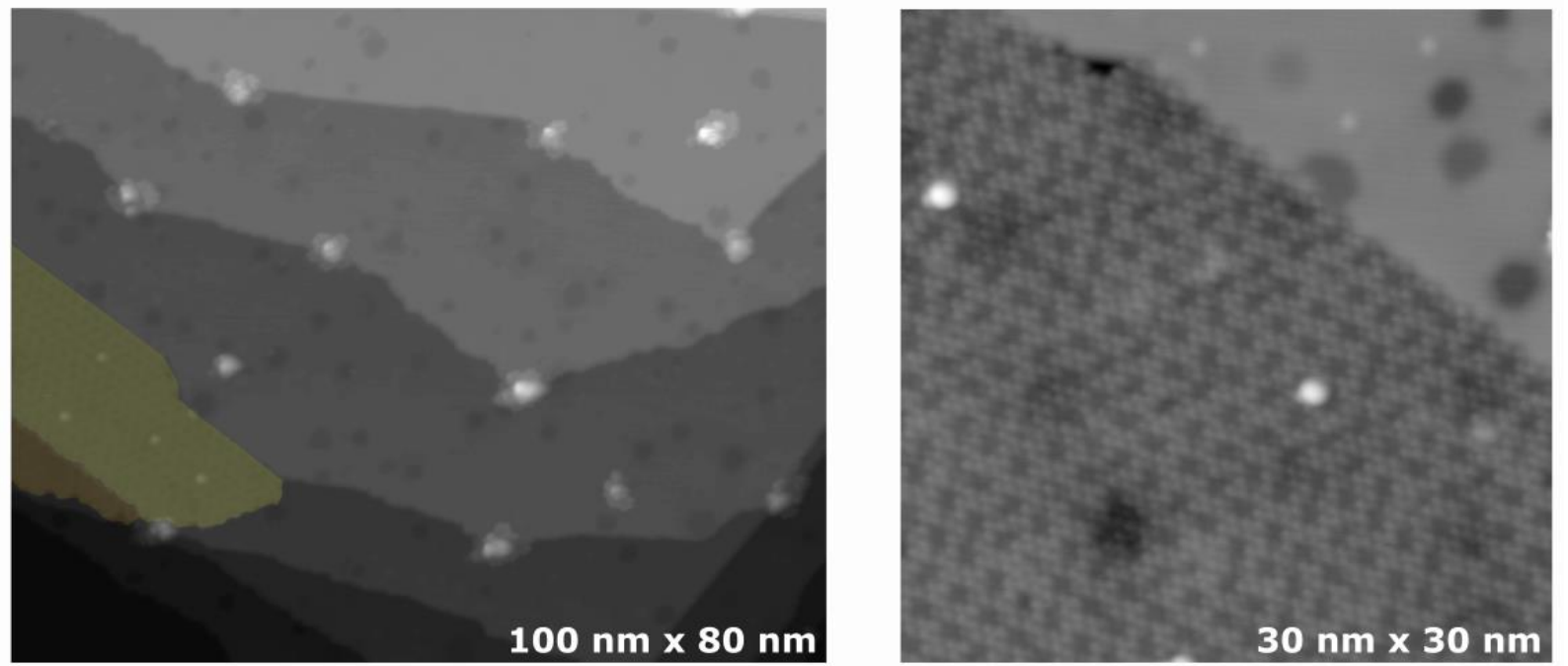

Fig. S1: STM images after deposition both molecules and $\mathrm{Cu}$ atoms. (left) Colored area indicates 2D molecule islands and (right) a zoom in in one of those islands. STM images conditions: $\mathrm{V}=100 \mathrm{mV}$ and $\mathrm{I}=10 \mathrm{pA}$. 


\section{Suppl. Info. S2: Atomic scale axle height}

The apparent height of a single and isolated $\mathrm{Cu}$ adatom on $\mathrm{Pb}(111)$ was compared with the apparent height of the atomic scale defect spontaneously formed at the $\mathrm{Au}(111) \mathrm{kink}$ dislocation. As presented in Fig. S2, they are equivalent in relative STM height (about 60 $\mathrm{pm})$ at $\mathrm{V}=500 \mathrm{mV}$ and $\mathrm{I}=10 \mathrm{pA}$.

\section{a. $\mathrm{Cu}$ adatoms on $\mathrm{Pb}(111)$}
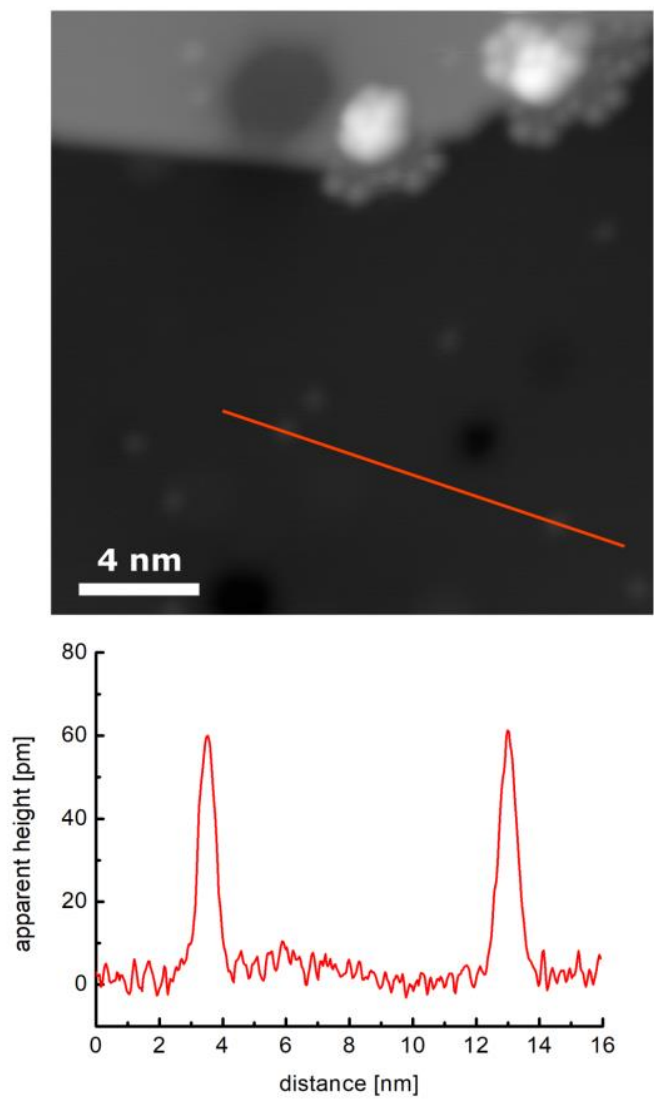

b. Au(111) surface
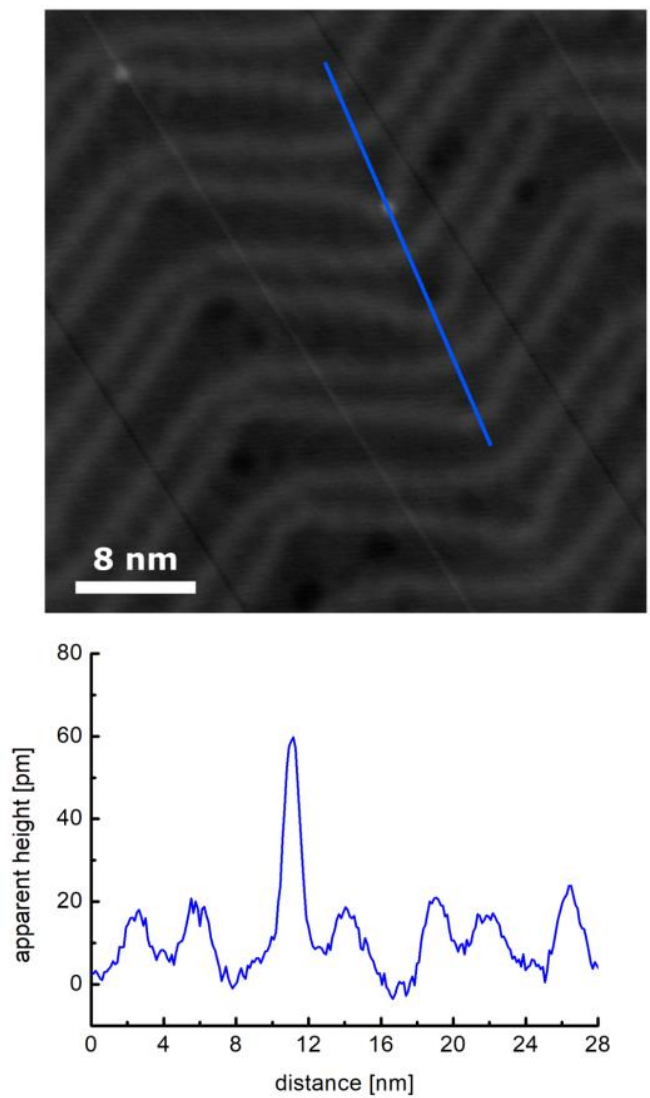

Fig. S2: STM constant current images of (a) $\mathrm{Cu}$ ad-atoms deposited on $\mathrm{Pb}(111)$ surface while keeping the $\mathrm{Pb}(111)$ surface at LHe temperature and (b) spontaneously formed atomic scale defect on the reconstructed Au(111) herringbone kink. Both STM images recorded with a $500 \mathrm{mV}$ bias voltage for a $10 \mathrm{pA}$ of tunneling current. 


\section{Suppl. Info. S3: Molecular mechanics calculations of the handle effect.}

Molecular mechanics calculations of the rotation potential energy barrier as a function of the collective rotation angle of a molecule-gear around its $\mathrm{Cu}$ ad-atom axle on the $\mathrm{Pb}(111)$ surface (Fig. S3.1). The total ground state energy of the molecular system was step by step optimized each $0.1^{\circ}$ for 2 turns and only one turn is presented. The semi-empirical ASED+ calculation technique ${ }^{1}$ was used taking into account the complete valence electronic of the system and the van der Waals interactions. The $\mathrm{Cu}$ ad-atom was supposed fixed in its hollow site on $\mathrm{Pb}(111)$ during the step by step optimization sequence. The rotation energy is given relative to the total electronic ground state of the system, including the $\mathrm{Pb}(111)$ slab. No periodic boundary conditions were used explaining the large $\mathrm{Pb}(111)$ considered in the ASED+ calculations. Notice that meshing the molecule handle with the molecule-gear is increasing the rotation barrier height from $0.22 \mathrm{eV}$ (Fig. S3.1) to almost $1 \mathrm{eV}$ explaining the stabilization effect of the handle molecule.
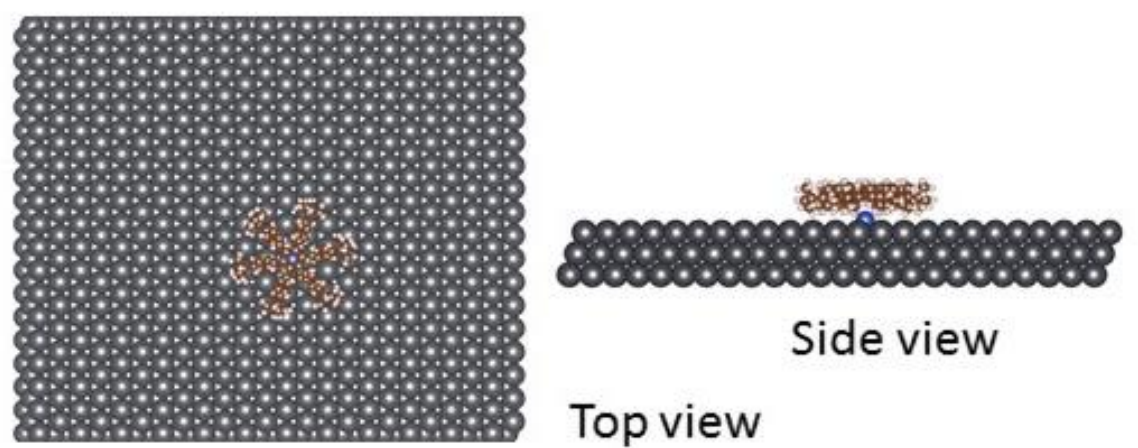

Top view

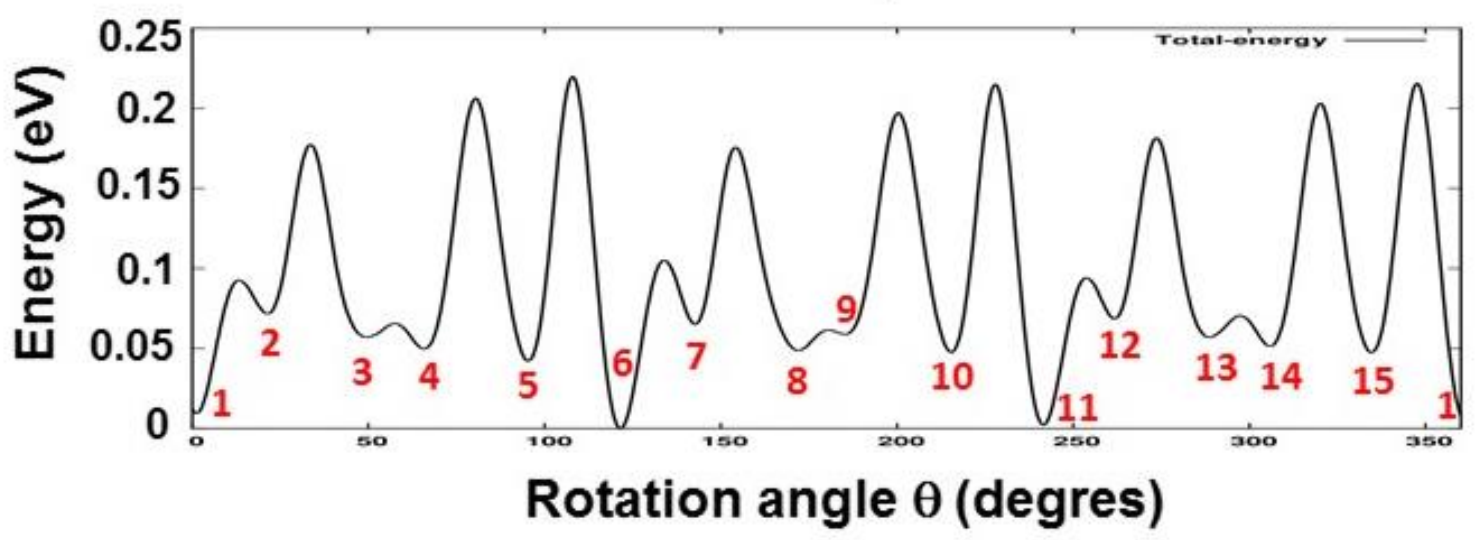

Fig. S3.1: Variation of the total energy of the $\mathrm{C}_{64} \mathrm{~N}_{2} \mathrm{H}_{76}$ molecule-gear mounted on a $\mathrm{Cu}$ adatom on $\mathrm{Pb}(111)$ as a function of its rotation angle and relative to its electronic ground state energy. All the possible 15 stable stations are indicated even if many of them cannot be reached because of possibility to de-engage the $\mathrm{C}_{64} \mathrm{~N}_{2} \mathrm{H}_{76}$ molecule from its $\mathrm{Cu}$ axle during an STM mechanical manipulation step (see Supp. Info. S4, Fig. S4.1 and the Fig. S3.2 below). 
Fig. S3.2 is presenting a 2D plot of the molecule-gear potential energy surface as a function the translation distance between the $\mathrm{C}_{64} \mathrm{~N}_{2} \mathrm{H}_{76}$ molecule center and the $\mathrm{Cu}$ ad-atom axle and as a function of the collective rotation angle $\theta$ of the molecule gear. It was calculated using ASED+ by a systematic optimization of the molecular structure per lateral step and keeping the $\mathrm{Cu}$ ad-atom in its hollow site on $\mathrm{Pb}(111)$.

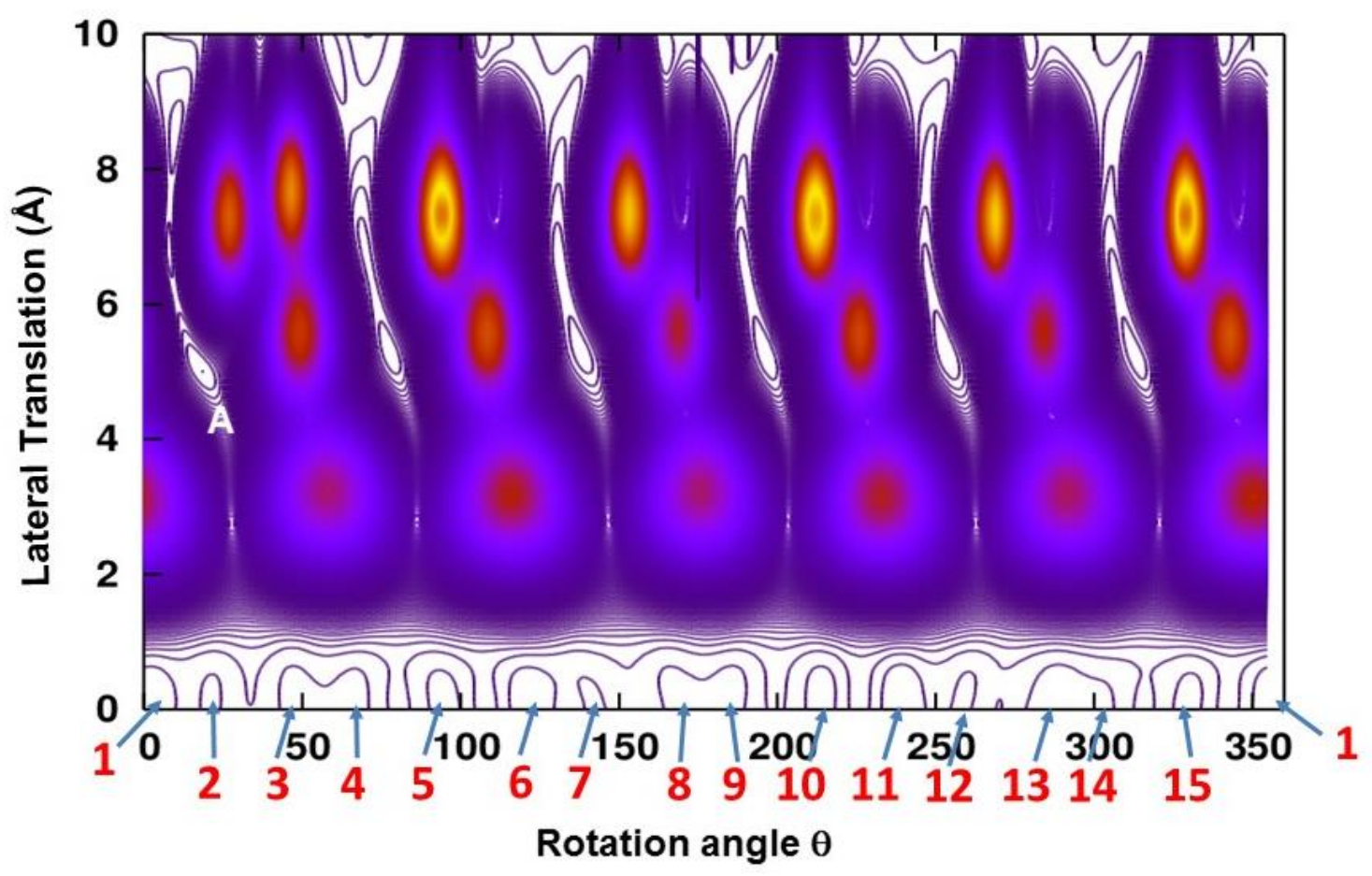

Fig. S3.2: The 2D plot of the potential energy surface of the rotation of the molecule-gear mounted on its $\mathrm{Cu}$ ad-atom axle in competition with a lateral translation of this molecule away from its $\mathrm{Cu}$ ad-atom. After a $1 \mathrm{~nm}$ distance away from the $\mathrm{Cu}$ ad-atom, the $\mathrm{C}_{64} \mathrm{~N}_{2} \mathrm{H}_{76}$ is almost free to diffuse on the $\mathrm{Pb}(111)$ surface. The 15 possible stable stations calculated in Fig. S3.1 are also indicated. A is indicating one of the 6 main dismounting paths for the $\mathrm{C}_{64} \mathrm{~N}_{2} \mathrm{H}_{76}$ molecule away for its $\mathrm{Cu}$ ad-atom axle.

(1) Ample, F.; Joachim, C., A Semi-Empirical Study of Polyacene Molecules Adsorbed on a $\mathrm{Cu}(110)$ Surface. Surf. Sci. 2006, 600, 3243-3251. 


\section{Suppl. Info. S4: Direct STM mechanical manipulation of a single molecule-gear}

We provide here two examples of LT-UHV-STM single molecule manipulation of a $\mathrm{C}_{64} \mathrm{~N}_{2} \mathrm{H}_{76}$ molecule-gear after its mounting on a single isolated $\mathrm{Cu}$ ad-atom on $\mathrm{Pd}(111)$. Those manipulations were both performed in a constant current manipulation mode to minimize the mechanical interactions between the STM tip apex end atom and the moleculegears. In Fig. S4.1, the molecule was dismounted from its $\mathrm{Cu}$ axle and in Fig. S4.2, the molecule-gear and its $\mathrm{Cu}$ axle are displaced together on the $\mathrm{Pb}(111)$ surface.

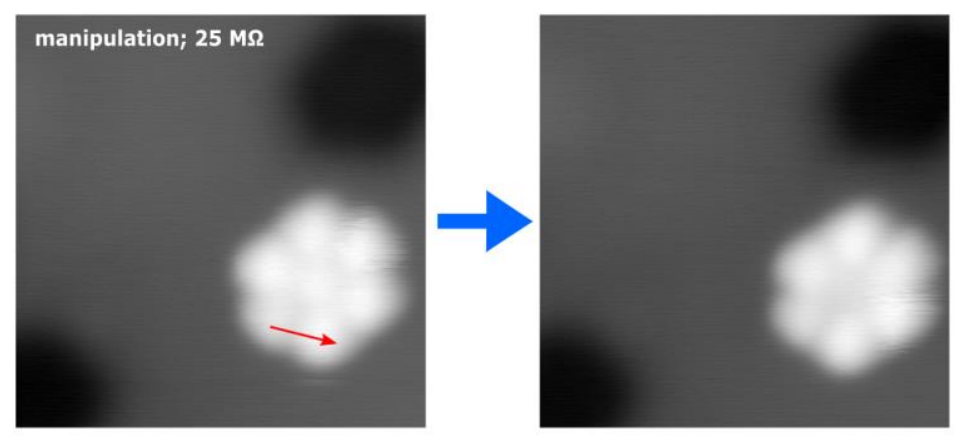

Fig. S4.1: Constant current STM lateral manipulation of a molecule-gear mounted of its $\mathrm{Cu}$ adatom axle on $\mathrm{Pb}(111)$. This manipulation was performed with a $25 \mathrm{M} \Omega$ tunnel junction resistance in a way to rotate the $\mathrm{C}_{64} \mathrm{~N}_{2} \mathrm{H}_{76}$ molecule around the $\mathrm{Cu}$ axle (the red arrow is indicating the exact tip apex trajectory during this experiment). After this manipulation sequence, the $\mathrm{C}_{64} \mathrm{~N}_{2} \mathrm{H}_{76}$ molecule was dismounted from its $\mathrm{Cu}$ axle and the $\mathrm{Cu}$ ad-atom is now in between 2 teeth (image size: $6 \mathrm{~nm} \times 6 \mathrm{~nm}$ ).

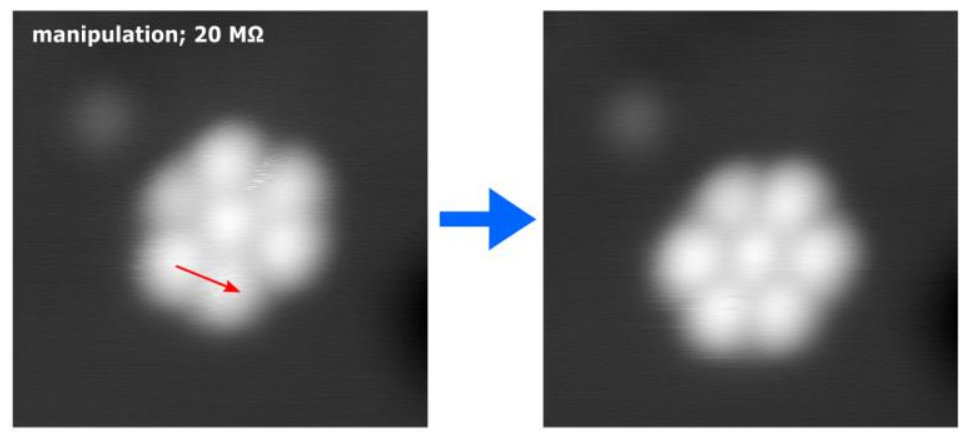

Fig. S4.2: Constant current STM lateral manipulation of a molecule-gear mounted of its $\mathrm{Cu}$ ad-atom axle on $\mathrm{Pb}(111)$. This manipulation was performed with a $20 \mathrm{M} \Omega$ tunnel junction resistance in a way to rotate the $\mathrm{C}_{64} \mathrm{~N}_{2} \mathrm{H}_{76}$ molecule around the $\mathrm{Cu}$ axle (the red arrow is indicating the exact tip apex trajectory during this experiment). After this manipulation sequence, the $\mathrm{C}_{64} \mathrm{~N}_{2} \mathrm{H}_{76}$ molecule and its $\mathrm{Cu}$ ad-atom axle were displaced together. This can be precisely measured taking into account the top left image isolated $\mathrm{Cu}$ ad-atom (image size: $5 \mathrm{~nm} \times 5 \mathrm{~nm})$. 


\section{Suppl. Info. S5: Rotational manipulation of a molecule-gear by voltage pulse}

Instead of mechanically manipulating the $\mathrm{C}_{64} \mathrm{~N}_{2} \mathrm{H}_{76}$ molecule, we have also attempted to bias voltage pulse the STM tip to induce a step by step rotation as presented below in Fig. S5.

I-V curve

a

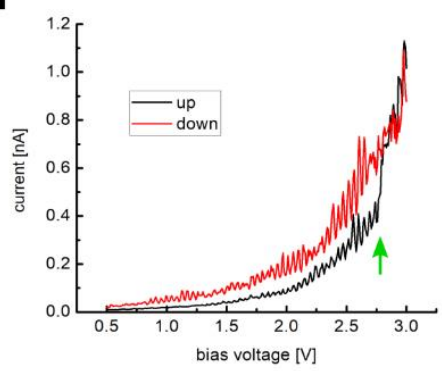

b

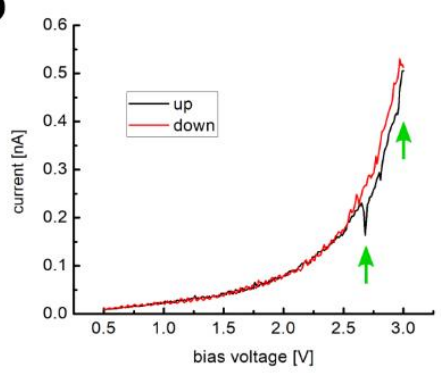

C

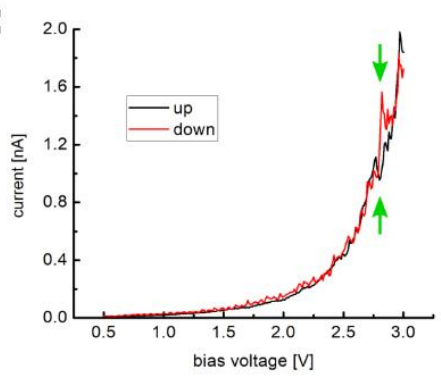

before
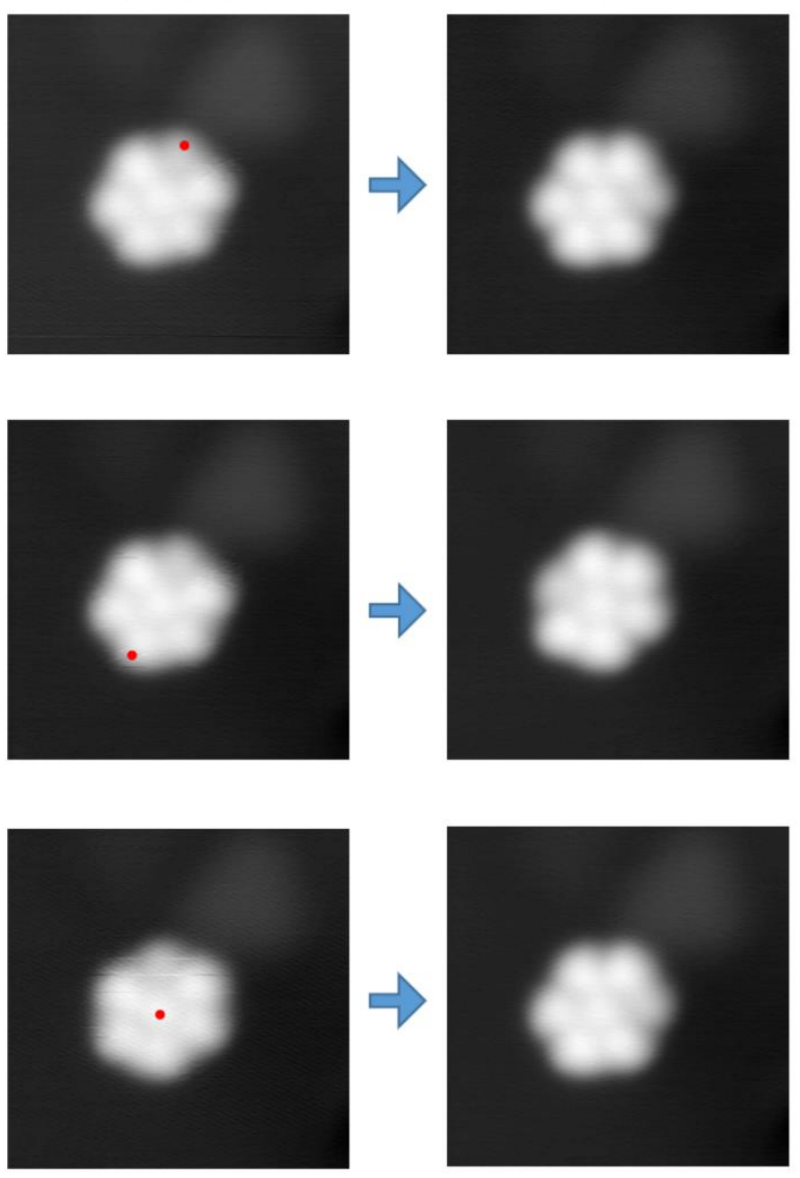

Fig. S5: The tip apex (red dots) was set on (a) the low apparent height tooth, (b) one of high apparent height teeth, and (c) center of molecule. When the bias voltage was reached around $2.7 \mathrm{~V}$, molecule conformation change was induced. That is, two methyl groups downward tert-butyl tooth was transformed into one methyl downward tooth (current jumps up when tip is located on the two methyl downward tooth as shown in the I-V curve a) and, at the same time, one of one methyl downward tooth was changed into two methyl downward tooth (current drops when tip is stayed on this transforming tooth as in the curve b). During this conformation switching, molecule also rotates undirectionally. Based on the I-V curve characteristics, it can be verified that $\mathbf{b}$ and $\mathbf{c}$ were triggered two times of conformation changes in contrast with only once transformation in $\mathbf{a}$ as indicated by green arrows. (Image size: $6 \mathrm{~nm}$ x $6 \mathrm{~nm})$. 


\section{Suppl. Info. S6: Movies}

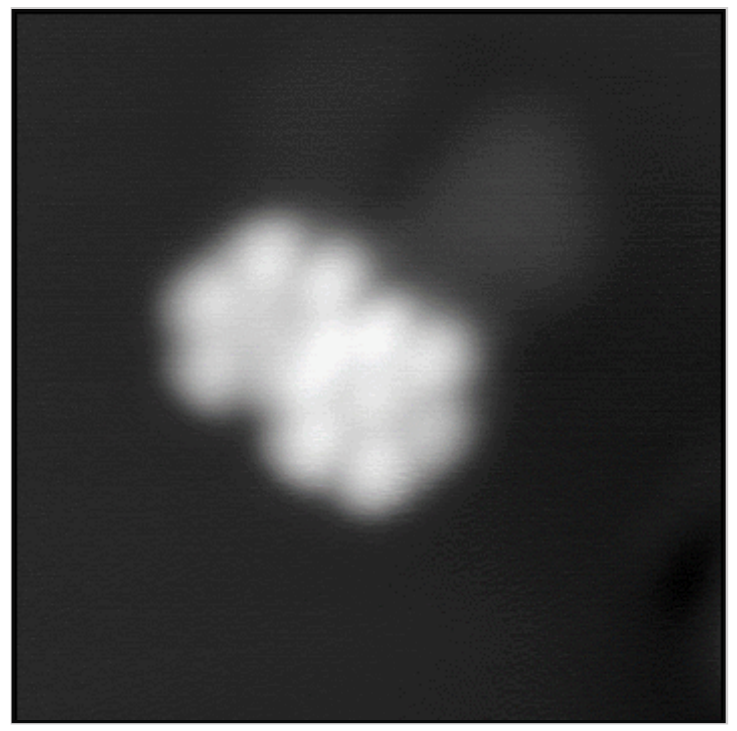

Movie S1: Full rotation of the $\mathrm{C}_{64} \mathrm{~N}_{2} \mathrm{H}_{76}$ molecule-gear using the $\mathrm{C}_{64} \mathrm{~N}_{2} \mathrm{H}_{76}$ molecule-handle by STM mechanical manipulation. This movie was displayed STM image per STM image in Fig. 2c we have here excluded the meta-stable stations ix and xii.

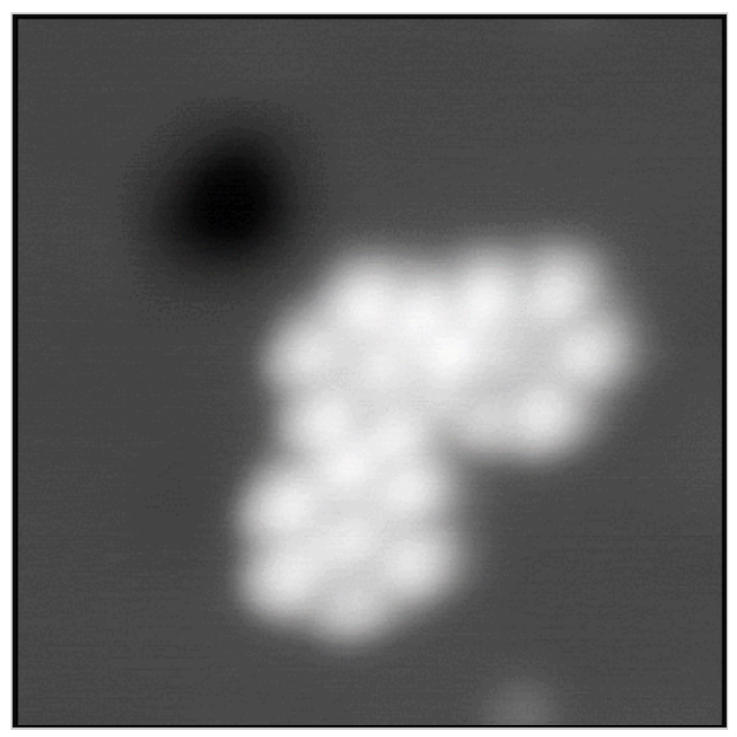

Movie S2: Transmission of gear rotation using STM mechanical manipulation along the $\mathrm{C}_{64} \mathrm{~N}_{2} \mathrm{H}_{76}$ molecule-gear train starting from the $\mathrm{C}_{64} \mathrm{~N}_{2} \mathrm{H}_{76}$ handle molecule. This movie was displayed STM image per STM image in Fig. 3. 


\section{Suppl. Info. S7: Ripping of the rotation transmission with only 2 molecule-gears}

Without an handle and to understand the observed experimental failure of the rotation transmission between 2 gears, the transmission of rotation from one gear to the next was simulated by molecular mechanics calculations using the semi-empirical ASED+ calculation technique taking into account the complete valence electronic structure of the system and the interaction between the 2 gears (van der Waals interactions, repulsive). Molecule-gear 1 was rotated only by pushing on one tooth at a time which normally triggers its collective rotation of angle $\Theta 1$. No need here to performed molecular dynamics calculations since the STM tip apex manipulation pushing on one teeth is experimentally very slow in time $(100 \mathrm{~ms})$. Molecule-gear 1 is interacting with molecule-gear 2. Both gear 1 and 2 are mounted on a single $\mathrm{Cu}$ ad-atom axle with a $\mathrm{Cu}-\mathrm{Cu}$ inter atomic distance equivalent to the experimental distance. In the ASED+ calculations, the 2 gears are also interacting with the $\mathrm{Pb}(111)$ surface with its atomic corrugation and electronic surface structure justifying the use of a semiempirical approach considering the number of atoms involved (see Fig. S7.1 insert).

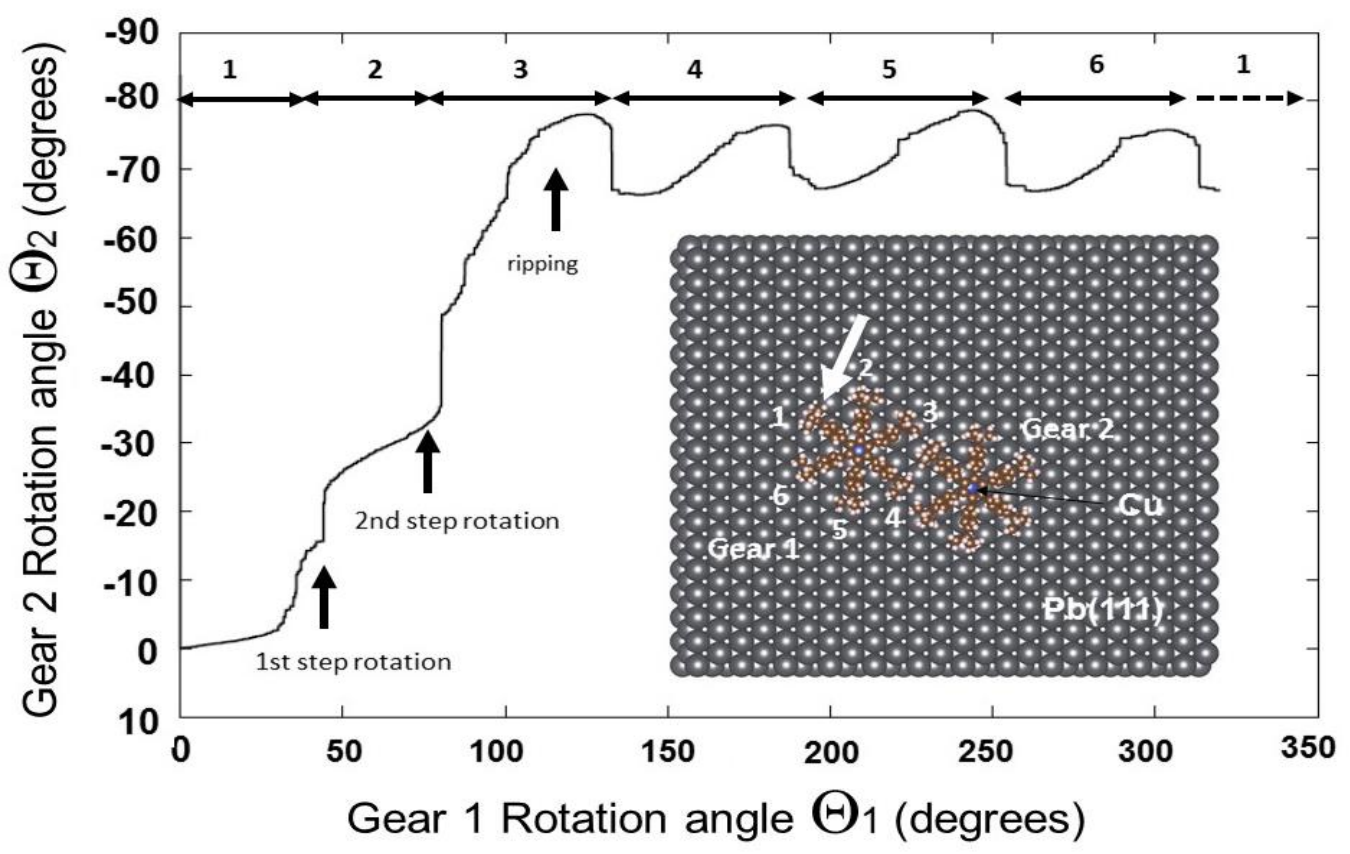

Fig. S7.1: Illustration of the blockage of the transmission of rotation from one molecule-gear to the next simulated using the ASED+ molecular mechanics method. In the insert, the white arrow is indicating the gear 1 tooth being pushed to reach $1^{\text {st }}$ step rotation. The ripping starts at pushing sequence 3 while the two gears are repealing each other (See Fig. S7.2 below).

The interactions between the 2 gears is via their respective teeth when engaged. They can be attractive or repulsive depending of the teeth inter atomic distances. Each teeth is also free to adopt any conformation, to rotate and deform depending on those interactions. The 
deformation angle of the pushed tooth was by step of 0.1 degrees. Then and at each step, the conformation of the complete molecule-gear dimers was optimized on the $\mathrm{Pb}(111)$ surface to find the best minimal energy conformation between (i) teeth deformations, (ii) lateral shift of the center of mass of each gear relative to its respective $\mathrm{Cu}$ axle, (iii) minimum energy conformation on the $\mathrm{Pb}(111)$ surface, (iv) the collective rotation of gear $1(\Theta 1)$ and (v) when occurring the transmission of rotation to gear $2(\Theta 2)$. Similar to experimental STM molecular manipulations, when a given tooth is no more the good one to trigger a collective rotation of gear 1, the push is performed on another tooth, the best one for triggering the next step rotation. Both gears are free to move laterally and to escape from their respective $\mathrm{Cu}$ axle.

As presented in the Fig. S7.1 above, the first rotation of gear 2 occurs after the $30^{\circ}$ rotation of gear 1 . But for gear 2 to reach its first complete $30^{\circ}$ rotation, gear 1 has almost to rotate $50^{\circ}$ because of the deformation of gear 2 teeth. Notice that those angles are calculated following the central phenyl rotation relative to its center of mass which is also changing. Then, a second $30^{\circ}$ rotation of gear 1 was triggered resulting is a $20^{\circ}$ rotation of gear 2 . Then, it was impossible to transmit more rotation to gear 2 whatever the tooth pushed on gear 1 and the rotation angle of gear 2 starts to oscillate back and forth by only $10^{\circ}$. The mechanics of this ripping and of the distance variation between the center of mass of the two gears was also extracted from the above molecular mechanics calculations. The resulting effect of pushing on the good tooth of gear 1 to trigger its rotation and then transmit a rotation motion to gear 2 is to bring step by step the two gears close together. Just before $\Theta 1=100^{\circ}$, the gear to gear repulsion effect occurs coming from the entangled teeth and the two gears are step by step de-centered from their $\mathrm{Cu}$ rotation axle. For $\Theta 1>250^{\circ}$, the teeth are no engaged enough together resulting in their deformation in a ripping oscillatory mechanics with no transmission of rotation. By calculations, we have tried different starting distances with the same resulting mechanical effect causes mainly by the flexibility of the teeth and the instability of the center of mass of each gear relative to its $\mathrm{Cu}$ respective axle. The only way we have found to master this ripping effect was to balance the interactions between the two engaged gears with an ancillary molecule not mounted on a $\mathrm{Cu}$ axle which in effect is restricting the center of mass motion of gear 1. 

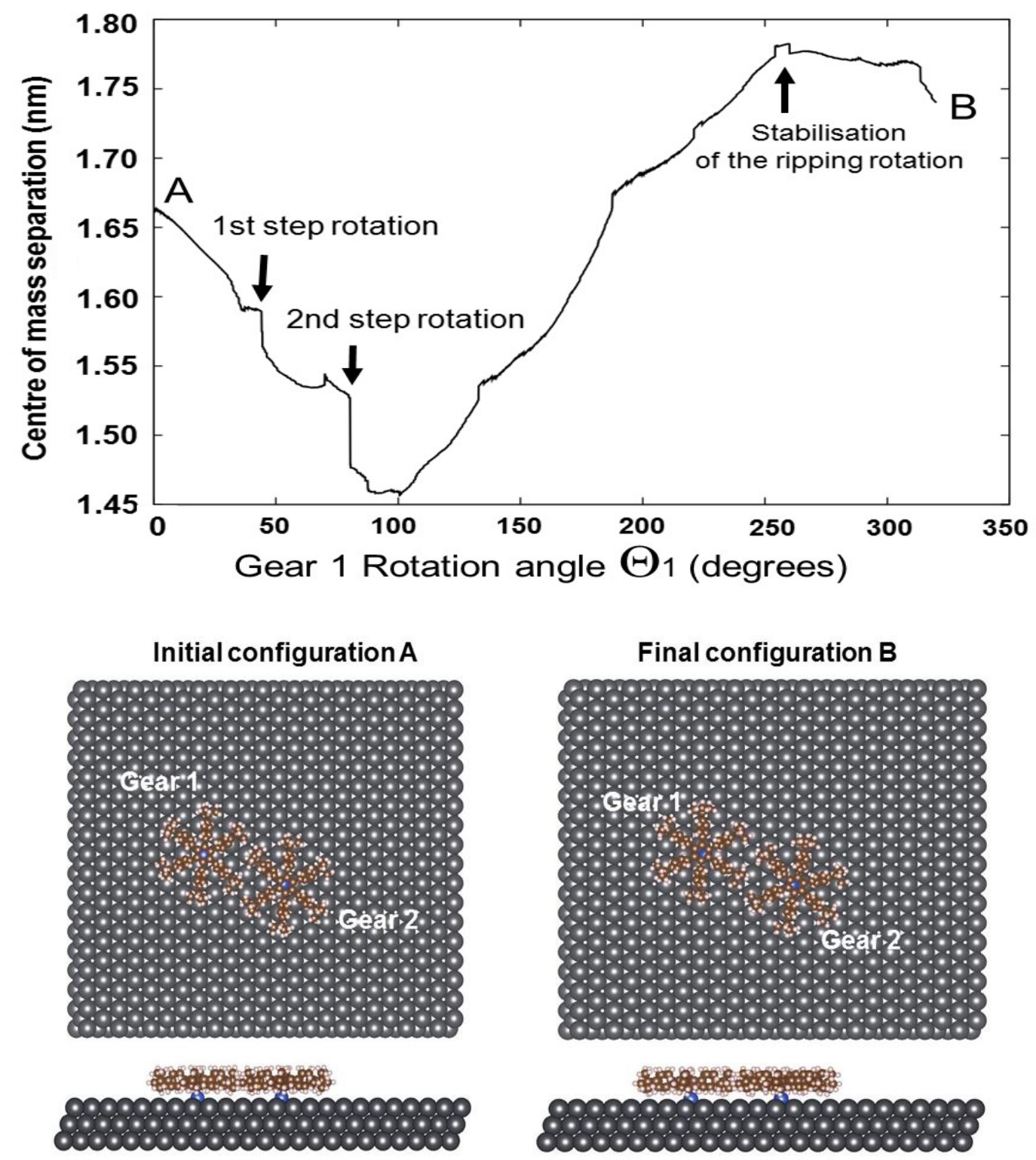

Fig. S7.2: Variations of the distance between the center of mass of the two molecule-gears as a function of the collective rotation angle $\Theta 1$ of gear 1 building up on the ground state potential energy surface of the complete molecular system represented in its initial optimized configuration A (left) and its final one B (right) for this calculation sequence. Simulating a pushing of a tooth of gear 1 increases the potential energy of the system. The resulting energy minimization can have for outcome a collective rotation of the gears or a deformation of the teeth. According also to fig. S7.1 and up to $100^{\circ}$, a collective rotation optimum path is found on the potential energy surface. Then for Q1 $>100^{\circ}$ and after a new push on the gear 1 tooth, teeth deformations is a better optimum energy path as compares to a transmission of rotation. 